\title{
Line bisectors and radial velocity jitter from SARG spectra ${ }^{\star}$
}

\author{
A. F. Martínez Fiorenzano ${ }^{1,2}$, R. G. Gratton ${ }^{2}$, S. Desidera ${ }^{2}$, R. Cosentino ${ }^{3,4}$, and M. Endl ${ }^{5}$ \\ 1 Dipartimento di Astronomia, Università di Padova, Vicolo dell'Osservatorio 2, 35122 Padova, Italy \\ e-mail: fiorenzano@pd.astro.it \\ 2 INAF - Osservatorio Astronomico di Padova, Vicolo dell'Osservatorio 5, 35122 Padova, Italy \\ 3 INAF - Osservatorio Astrofisico di Catania, Via S. Sofia 78, Catania, Italy \\ ${ }^{4}$ INAF - Centro Galileo Galilei, Calle Alvarez de Abreu 70, 38700 Santa Cruz de La Palma (TF), Spain \\ 5 McDonald Observatory, The University of Texas at Austin, Austin, TX 78712, USA
}

Received 16 February 2005 / Accepted 11 July 2005

\section{ABSTRACT}

We present an analysis of spectral line bisector variations for a few stars observed in the SARG high precision radial velocity planet survey, and discuss their relationship with differential radial velocities. The spectra we consider are the same used for determining radial velocities. The iodine cell lines employed in the measurement of radial velocities were removed before bisector analysis. The line bisectors were then computed from average absorption profiles obtained by cross correlation of the stellar spectra with a mask made from suitable lines of a solar catalog. Bisector velocity spans were then determined: errors in these quantities compare well with theoretical expectations based on resolution, $S / N$ and line shape. The plot of bisector velocity span against radial velocity was studied to search for correlations between line asymmetries and radial velocity variations. A correlation was seen for HD 166435 due to stellar activity, and for HD 8071B due to spectral contamination by the companion. No correlation was seen for $51 \mathrm{Peg}$ and $\rho \mathrm{CrB}$, stars hosting planets. We conclude that this technique may be useful to separate radial velocity variations due to barycenter motion from spurious signals in spectra acquired with the iodine cell.

Key words. stars: atmospheres - stars: activity - stars: planetary systems - techniques: spectroscopic - techniques: radial velocities line: profiles

\section{Introduction}

The study of activity jitter is mandatory in the search for exoplanets using the radial velocity $(R V)$ technique, because it represents an important source of noise and a proper analysis is necessary to discard false alarms (e.g., HD 166435: Queloz et al. 2001; HD 219542B: Desidera et al. 2003, 2004a). The differential $R V$ variations induced by stellar activity are due to changes in the profile of spectral lines caused by the presence of spots and/or the alteration of the granulation pattern in active regions (Saar \& Donahue 1997; Hatzes 2002; Saar 2003; Kürster et al. 2003). The activity jitter of a star may be predicted by means of statistical relations from its chromospheric emission, rotational velocity or amplitude of photometric variations (Saar et al. 1998; Paulson et al. 2004). Simultaneous determination of $R V$, chromospheric emission and/or photometry is even more powerful in disentangling the origin of the observed $R V$ variations Keplerian vs. stellar activity. However these techniques cannot be considered as a direct measurement

* Based on observations made with the Italian Telescopio Nazionale Galileo (TNG) operated on the island of La Palma by the Centro Galileo Galilei of INAF (Istituto Nazionale di Astrofisica) at the Spanish Observatorio del Roque del los Muchachos of the Instituto de Astrofísica de Canarias. of the alterations of the spectral line profiles that are the origin of the spurious $R V$ variations.

This type of study can be carried out by considering variations of line bisectors, that may be thought of as direct measure of activity jitter (Queloz et al. 2001) through the evidence of variations of the asymmetries which appear in spectral lines. Line bisectors are employed in the analysis of asymmetries (Gray 1982, 1988) due to stellar atmospheric phenomena like granulation (Dravins et al. 1981), turbulence or pulsation that sometimes are responsible for the $R V$ variations observed in stars.

The reason for studying simultaneously the variations of the $R V$ and of the (average) line bisector of a star is to determine if any variation of the measured $R V \mathrm{~s}$ are caused by a center of mass motion due to celestial bodies orbiting a star (see Queloz et al. 2001; and Desidera et al. 2003).

The analysis of spectral line asymmetries also has an important application for the follow-up of transit surveys. An eclipsing binary in a hierarchical triple system and a transiting planetary companion might produce similar photometric signature; but very different line bisector variations, allowing one to disentangle the characteristics of the system (Torres et al. 2004, 2005). In addition, asymmetries of spectral lines may arise due to contamination of the spectrum by a nearby star. This point 
is of particular relevance for the targets we are studying in the SARG survey (Desidera et al. 2004b), because by design all of them are visual binaries. In this case a companion near the line of sight may contaminate the spectral features of the star being observed. Finally, spurious line profile asymmetries may be due to instrumental causes, e.g., non symmetric illumination of the slit.

Line bisector variations may be studied quite easily in spectra acquired using fibers (see e.g., Queloz et al. 2001). The fibers provide a constant, roughly symmetric illumination of the slit. Furthermore, spectra are generally acquired with simultaneous wavelength calibration lamps, rather than imprinting absorption cell features on the stellar spectral lines. No attempt has been made to our knowledge to study line bisectors on spectra obtained through an iodine cell. One disadvantage is the necessity to remove the iodine lines from the stellar spectra; but on the other hand, the iodine lines allow a fine wavelength calibration and the possibility of monitoring the instrumental profile.

In this paper we present such an attempt; line bisectors and radial velocities are determined for SARG stellar spectra, to study possible trends between spectral line shapes (line bisectors) and $R V \mathrm{~s}$.

This paper describes the procedure followed to handle the spectra, to remove iodine lines, the construction of a mask made from selected lines of a solar catalogue, the cross correlation function (CCF) computed between mask and stellar spectra, the determination of the line bisector and the calculation of the bisector velocity span. We present results obtained for five stars: we found a clear correlation between the line bisector velocity span and $R V$ for HD 166435, likely due to stellar activity, consistent with previous published work by Queloz et al. (2001). A similar correlation was found for HD 8071B due to contamination by light from the companion. We did not find any correlation for stars known to host planets like 51 Peg and $\rho \mathrm{CrB}$, and the false alarm (inconclusive) case of HD 219542B.

In the next section we describe some aspects of the observations; Sect. 3 explains the procedure employed in the analysis with results presented in Sect. 4. The last two sections are devoted to a discussion of the error analysis and conclusions.

\section{Observations and data reduction}

The data discussed in the present paper are part of the $R V$ survey aimed to find planets around stars in wide binaries (Desidera et al. 2004b), ongoing at TNG using the high resolution spectrograph SARG (Gratton et al. 2001). The spectra have a resolution of $R \sim 150000$, covering the spectral range $4580 \AA-7900 \AA$ in 55 echelle orders, with $S / N$ values in the range 70-400 and were taken from September 2000 until August 2004. Slit width was set at 0.27", much smaller than the typical seeing Full Width Half Maximum (FWHM). Furthermore, an autoguider system, viewing the slit by means of a detector with its wavelength response peaked at the wavelength of the iodine cell lines, was employed, keeping the instrumental profile stable and fairly independent of illumination effects. Guiding was generally done using the image of the binary companion on the slit viewer.
Data reduction was performed in a standard way using IRAF $^{1}$. High precision $R V \mathrm{~s}$ were measured on these spectra with the AUSTRAL code (Endl et al. 2000) as described in Desidera et al. (2003).

The iodine cell technique includes the acquisition of spectra from a featureless source (a fast rotating B-star or the flat field lamp) taken with the iodine cell inserted in the optical path (Butler et al. 1996). This kind of spectra is necessary only for the deconvolution of stellar templates (taken without the cell), but they were acquired also in most of the observing nights of our survey to monitor instrument performances. These featureless source spectra were used to remove the iodine lines from the science spectra, as explained in the next section.

\section{Data analysis}

\subsection{Handling of the spectra}

The iodine lines, superimposed on the stellar spectrum for wavelength calibration and $R V$ determination, were eliminated because the line bisector is intended to study the asymmetries of the stellar spectral lines alone. For this task, spectra of fast rotating ( $V \sin i \geq 200 \mathrm{~km} \mathrm{~s}^{-1}$ ) B-stars were employed. The analysis was made only for the wavelength range where the iodine lines appear in the spectra (5036 $\AA-6108 \AA$ ) along 21 orders. We used only this spectral region because there the wavelength calibration is more accurate, because of the iodine lines themselves.

To handle the spectra, each order was divided in 7 pieces of 500 spectral points each, corresponding to a wavelength width of $\sim 10 \AA$, overlapped by 60 points $(\sim 1 \AA)$ to eventually recover any absorption lying at the edges of the chunks. This procedure of cutting the spectra in pieces was clearly advantageous, while the division of complete orders displayed not optimal results, related to errors in the wavelength calibration of the spectra (see Fig. 1). A cross correlation computed between the spectral chunks of the B-star and the program star determined the offset in wavelength between the iodine lines common to both spectra. The B-star spectrum was adjusted using a Hermite spline interpolation (INTEP, see Hill 1982) to the wavelength scale defined by the program star spectrum after application of the appropriate offset and finally divided to the program star spectrum (see Fig. 2; details will appear elsewhere: Martínez Fiorenzano 2005). The success of this procedure depends on the stability of the instrumental profile over time, since the B-star spectra were usually acquired at the beginning or the end of each night.

In a few cases, the cross correlation procedure used to determine the wavelength offset of chunks did not provide a reasonable value; in these cases the division of the star flux by the B-star flux added noise rather than removing the iodine lines. These noisy chunks were rejected from further analysis.

Spikes due to cosmic rays or hot pixels were removed by replacing the spectral values within them with the averaged flux of adjacent spectral points. This procedure was only

\footnotetext{
1 IRAF is distributed by the National Optical Observatory, which is operated by the Association of Universities for research in Astronomy, Inc., under contract with the National Science Fundation.
} 


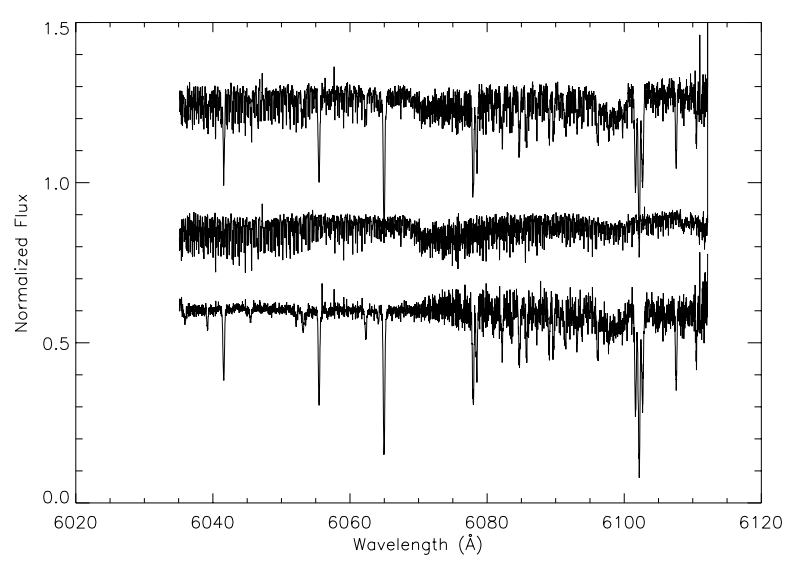

Fig. 1. Portion of a spectrum from HD 166435. The top spectrum corresponds to the program star with iodine lines, in the middle is the spectrum of the B-star with iodine lines and on the bottom is the program star spectrum after the division by the B-star spectrum. The division was computed for the whole order: the poor result is due to errors in the wavelength calibration along one order. In this case the iodine lines are better removed in the left half than in the right half of the order.

applied to those cases where spikes occurred far from the line centers. In the very rare cases where the spikes occurred near the centers of the lines used in our analysis, possibly affecting the line bisector analysis described below, the relevant chunks were simply removed from further analysis, from all spectra of the same star.

\subsection{The cross correlation function (CCF)}

\subsubsection{The solar catalogue and line selection for the mask}

The list of spectral lines by Moore et al. (1966) was used to prepare the spectral mask needed for the computation of the CCF. A preliminary line list was obtained by selecting those lines that do not have possible contaminants (wavelength separation $\gtrsim 0.1 \AA$ ), and have reduced width between 3 and $30 \mathrm{~F}$. F (Fraunhofer) is defined as the dimensionless quantity $\Delta \lambda / \lambda \times 10^{6}$, where $\Delta \lambda$ is the equivalent width (see Moore et al. 1966). The range of reduced width chosen here corresponds to a range of central line depths from 0.14 to 0.75 in continuum units (in the solar spectrum). The CCFs we derived thus represent average profile for lines of intermediate strength.

Once a preliminary list of lines was determined for the wavelength range of interest (5036 $\AA$ to $6108 \AA$ ), a further selection was made by inspecting the "The Sacramento Peak Atlas of the Solar Flux Spectrum" (Beckers et al. 1976). Lines were labeled according to their appearance: "y" (very good): sharp, clear, with weak wings; "y:" (good): clear but near other lines altering their wings; "?" (not very good): with small blends or strong wings. Many other lines, blended or too close to other spectral lines, were removed from the list at this step.

The mask used to compute the CCF is a sum of $\delta$-function profiles corresponding to 1 for the line wavelength, with a base of two (spectral) points, and 0 elsewhere. The extensive line
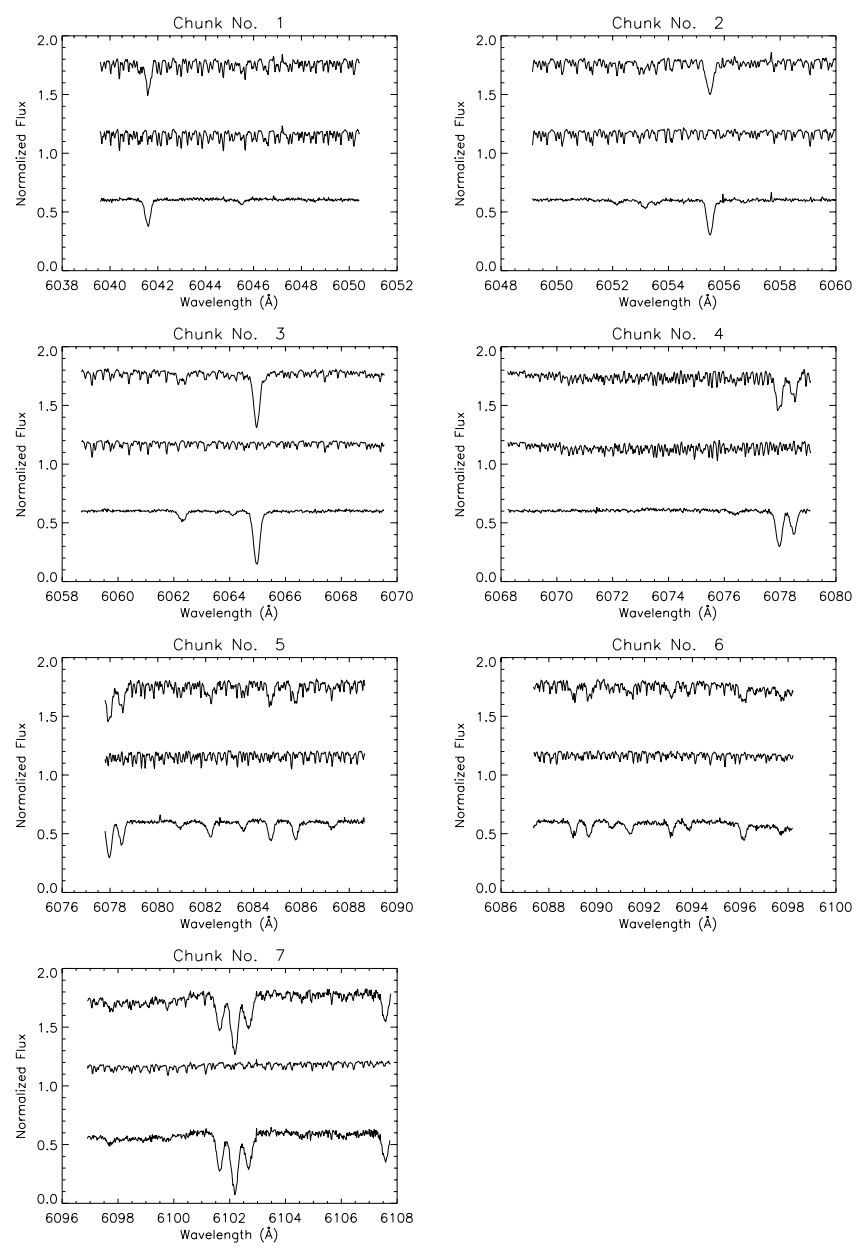

Fig. 2. An order of a spectrum of HD 166435 (wavelength range $6039 \AA-6107 \AA$ ) shown in Fig. 1. The order has been divided into 7 chunks, as described in the text. The spectra are shifted arbitrarily in flux to show: on top the stellar spectra with iodine lines, in the middle the B-star spectra (adjusted to the wavelength offset) with the iodine lines and on the bottom the clean stellar spectra after dividing the program star spectrum by the B-star spectrum.

list (including very good, good and not very good lines: a total of about 300 lines) served to determine the $R V$; this gave the centroid value of the average absorption profile obtained by the CCF. Once this point was established, which helps to properly locate the profiles for the addition, a new computation of the CCF was performed only with the very good lines to obtain an improved average profile, used to calculate reliable average line bisectors (see Fig. 3).

\subsubsection{The cross correlation and addition of profiles}

A cross correlation between the mask and the stellar spectrum was computed for each chunk; the addition of all these cross correlations gave the average profile. Due to the relatively low $S / N$ of some spectra, use of the average of many lines is appropriate for this study (the variations of line bisectors with time). On the other hand, the actual line bisector depends on the line depth, so that our "average" line bisector does not rigorously correspond to the line bisector of a line with similar depth of 

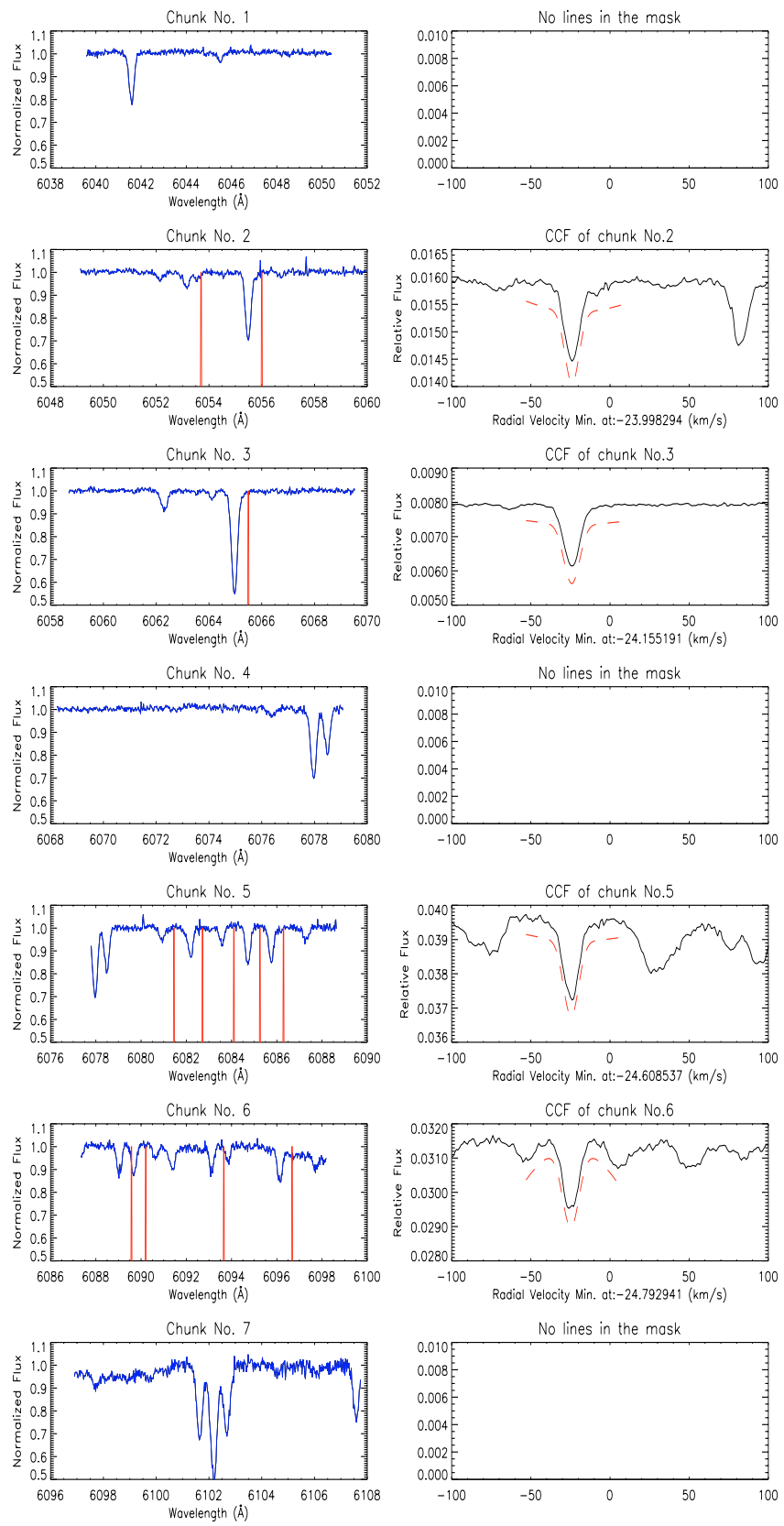

Fig. 3. An order of a spectrum of HD 166435. The left column shows individual chunks of the spectrum (blue lines), after removal of the iodine cell lines (see Fig. 1). Overimposed (as red lines) is the mask used for the determination of the CCF (only lines classified as very good are shown here). It is made of a sum of $\delta$-functions centered on the rest wavelengths of the selected lines. Note the wavelength shifts between the stellar lines and the mask peaks due to the non-zero radial velocity of the star. The CCF for individual chunks computed by cross-correlating the spectra with the masks given on the left panels are shown in the right panels. The dashed line, shifted slightly below the profiles for clarity, represent the Gaussian fit computed to determine the local minimum of the CCF for each chunk. There are no CCF profiles for chunks 1,4 and 7 due to the lack of suitable lines for the mask in these wavelength ranges.

the CCF. Therefore, the use of our average profile would be misleading for some scientific goals such as the study of convection in stellar atmospheres.
Due to different illumination of the CCD, each chunk along an order has different flux values, those close to the center of the orders being more luminous and yielding then results of higher $S / N$. To account for this, before summing the individual cross correlations, each of them was multiplied by an appropriate weight, proportional to the instrumental flux at the center of the chunk.

Once adjusted to a common reference frame, which may be thought of as a centering procedure, and multiplied by its weight, all CCF profiles were added to get the final profile for the spectrum. This was normalized to determine the finally adopted average line profiles using the reference continuum determined in the IRAF reduction. This was obtained by interpolating a polynome throughout the spectra with a suitable clipping rejection procedure. Note that this reference continuum may contain significant errors, so that there may be points in the normalized CCFs that are well above unity. However, we kept our procedures strictly uniform throughout the analysis of different spectra; we then expect that these errors in the location of the reference continuum mainly affect absolute profiles and bisector estimates, but only marginally their spectrum-tospectrum variations, which are of interest for the present discussion.

\subsection{The line bisector calculation}

The bisector of an absorption line is the middle point of the horizontal segment connecting points on the left and right sides of the profile with the same flux level. The line bisector is obtained by combining bisector points ranging from the core toward the wings of the line.

For the bisector determination, it is necessary to adjust the ordinate axis of the profile to a convenient scale and to determine the values in velocity belonging to the left and right points for a given flux value. This is accomplished by interpolating (using INTEP, see Hill 1982) the absorption profile to the desired scale (details will appear in Martínez Fiorenzano 2005).

\subsection{The bisector velocity span}

To quantify the asymmetry of the spectral lines and look for correlation with $R V$ it is useful to introduce the bisector velocity span (Toner \& Gray 1988). This is determined by considering a top zone near the wings and a bottom zone close to the core of the lines, which represent interesting regions to study the velocity given by the bisector (see Fig. 4). The difference of the average values of velocities in the top and bottom zones, $V_{\mathrm{T}}$ and $V_{\mathrm{B}}$ respectively, determine the bisector velocity span (BVS for short).

The location of the top and bottom zones, as well as their width $\Delta F$, were determined as percentages along the absorption profiles. They were defined using as templates the spectra of HD 166435; the same zones were then employed in the study of the other objects to be consistent in the analysis procedure.

The Spearman nonparametric rank correlation coefficient was computed to establish the significance of the linear correlation between $\mathrm{BVS}$ and $R V$ for each star. 

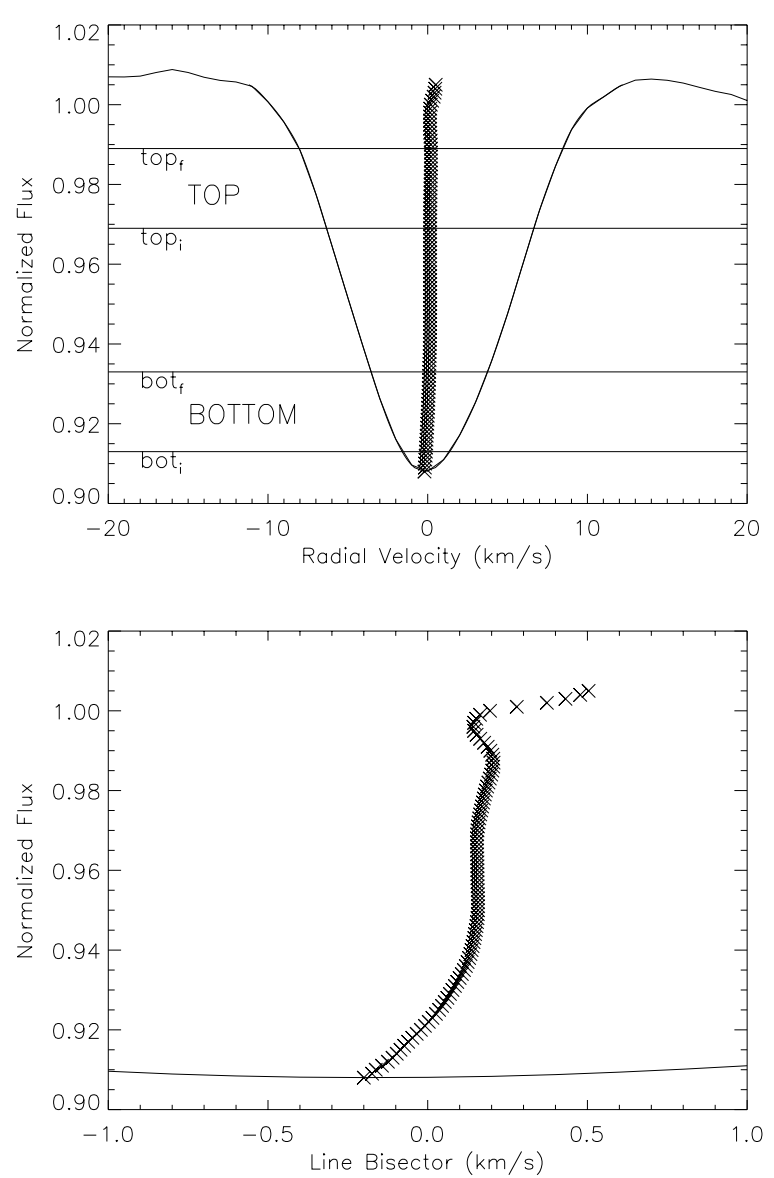

Fig. 4. Spectrum of HD 166435. In the top panel we show the normalized cross correlation profile, the line bisector, the top and bottom zones (both with $\Delta F=0.02 ; \Delta F=$ top $_{\mathrm{f}}-$ top $_{\mathrm{i}}=$ bot $_{\mathrm{f}}-$ bot $_{\mathrm{i}}$ ). In the bottom panel we show a zoom of the profile with the $R V$ scale increased to better display the asymmetries of the line bisector.

\subsection{Error determination}

The expression given by Gray (1983) for the bisector error due to photometric error was employed to establish the error for the bisector velocity span. The photon noise error, determined for the specific case of SARG using Gray's equation, yields the expression:

$\delta V=\left(\frac{S}{N}\right)^{-1}\left(2 n F \frac{\Delta F}{x} \frac{\mathrm{d} F}{\mathrm{~d} V}\right)^{-1 / 2}$,

where $S / N$ is the signal-to-noise of the spectrum, including the contribution of the B-star spectrum and it is given by: $\left((N / S)_{\text {star }}^{2}+(N / S)_{\mathrm{B}-\mathrm{star}}^{2}\right)^{-1 / 2}$, with values between $65-300$. The $\mathrm{B}$-star spectra employed in the analysis were acquired on the same observing nights of the target spectra and have typical $S / N$ values $\gtrsim 250$. In Eq. (1) $n$ is the number of lines in the mask for the CCF; $F$ is the central flux of the zone of analysis; $\Delta F$ is the interval of flux determining the zone; $x$ is the scale of the spectrum based on the linear dispersion of the instrument ( $1.04 \mathrm{~km} \mathrm{~s}^{-1} \mathrm{pix}^{-1}$ for $\mathrm{SARG}$ ) and $\mathrm{d} F / \mathrm{d} V$ gives the slope of the profile in the zone being analyzed (see Fig. 4).

Error bars for the BVS were then determined by adding quadratically the errors for the top and bottom zones.
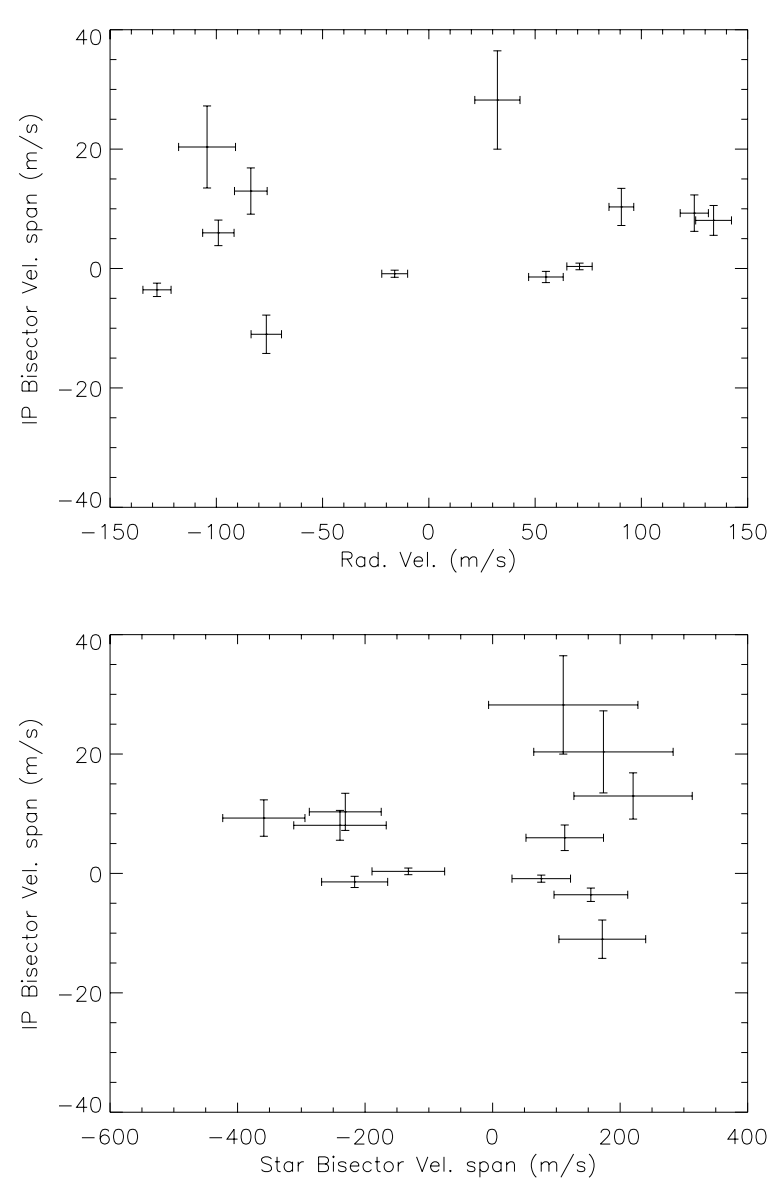

Fig. 5. Instrumental profile (IP) analysis. Top panel: plot of IP BVS vs. $R V \mathrm{~s}$ of HD 166435. Bottom panel: plot of IP BVS vs. BVS of HD 166435. No evidence of trends were found.

\subsection{Instrument Profile asymmetries}

Line bisector variations may arise from variations of the stellar spectra as well as from variations of the instrumental profile (IP). We studied the IP determined by AUSTRAL as part of the $R V$ determination and computed line bisectors for it as for stellar spectra.

Generally the line bisector variations of IP are significant, but they are typically much lower than the errors in the line bisectors determined from the stellar spectra. Corrections due to asymmetries of line profiles require complex convolution, which should take into account the real stellar profile. Actually, since the IP is much narrower than the intrinsic stellar line profile (the case of SARG spectra), corrections due to IPs are much smaller than the IP asymmetries themselves; therefore we did not apply corrections to the stellar BVS.

In Fig. 5 we show the run of the BVS of the IP with $R V$ and the IP BVS with the stellar BVS in the analysis of HD 166435. No clear trend could be discerned.

\section{Results}

\section{1. $H D 166435$}

The dwarf star HD 166435, of spectral type G0 and visual magnitude $m_{V}=6.8$, shows evidence of $R V$ variations, photometric 
Table 1. Bisector velocity span from spectra of HD 166435. 53 lines were employed in the mask for the CCF.

\begin{tabular}{cccc}
\hline \hline JD - 2450000 & $\begin{array}{c}\left(V_{\mathrm{T}}-V_{\mathrm{B}}\right) \\
\mathrm{m} \mathrm{s}^{-1}\end{array}$ & $\begin{array}{c}V_{r} \\
\mathrm{~m} \mathrm{~s}^{-1}\end{array}$ & $S / N$ \\
\hline 2775.65 & $-231.1 \pm 56.4$ & $90.6 \pm 5.8$ & 164 \\
2776.68 & $154.1 \pm 57.8$ & $-127.9 \pm 6.6$ & 156 \\
2809.62 & $-216.4 \pm 51.8$ & $55.1 \pm 8.2$ & 178 \\
2818.62 & $173.8 \pm 109.3$ & $-104.4 \pm 13.4$ & 86 \\
2860.40 & $220.3 \pm 92.8$ & $-83.8 \pm 7.7$ & 98 \\
2861.42 & $110.8 \pm 117.1$ & $32.3 \pm 10.6$ & 81 \\
2862.42 & $-358.8 \pm 64.5$ & $124.9 \pm 6.6$ & 140 \\
2891.37 & $172.0 \pm 68.1$ & $-76.5 \pm 7.2$ & 138 \\
2892.35 & $-239.4 \pm 72.5$ & $134.0 \pm 8.4$ & 126 \\
3129.74 & $113.1 \pm 60.8$ & $-99.0 \pm 7.4$ & 149 \\
3130.73 & $76.3 \pm 45.9$ & $-16.1 \pm 6.1$ & 201 \\
3131.74 & $-132.2 \pm 57.0$ & $70.9 \pm 6.0$ & 164 \\
\hline
\end{tabular}

variability and magnetic activity. Furthermore, previous analysis of the variation of the line bisectors revealed a correlation between $R V$ and line bisector orientation (Queloz et al. 2001).

The large amplitude of the activity-induced variations and their stability make this star an ideal target to test new procedures like those presented in this paper.

Twelve spectra of HD 166435 were acquired between May 2003 and May 2004. The line bisectors, derived from the spectra, were used to study the correlation between the BVS and the $R V \mathrm{~s}$. The plot of BVS against $R V\left(\left(V_{\mathrm{T}}-V_{\mathrm{B}}\right)\right.$ vs. $\left.V_{r}\right)$ was fitted to a straight line.

In principle, the location and extension of top and bottom zones of the line profiles used in the derivation of the BVS are arbitrary. In order to optimize detection of variations of the BVS correlated with $R V$ variations, we maximized the $m / \sigma$ ratio (where $m$ is the slope of the straight line fitting of BVS against $R V$, and $\sigma$ its uncertainty) over different choices of the location and amplitude of the line profile regions used to derive the BVS. The $m / \sigma$ ratio is an indicator of the significance of the linear term in the straight line fitting.

The top and bottom zones were determined according to the relative absorption percentages in which the highest significance was found: top centered at $25 \%$ of the maximum absorption, bottom at $87 \%$; in both cases the width $\Delta F$ was of $25 \%$. For consistency, these percentage values were then considered for the analysis of all the stars.

The upper panel of Fig. 6 displays the plot of BVS against $R V$; superimposed is the best fitting straight line, with:

$\left(V_{\mathrm{T}}-V_{\mathrm{B}}\right)=(-1.98 \pm 0.21) \times V_{r}+(-29.03 \pm 18.45)$.

The rank correlation coefficient is -0.89 and the significance is of $99.99 \%$. The lower panel of the same figure shows the line bisectors computed for all spectra of HD 166435, corrected for their $R V$ and plotted on a common reference frame. Values of bisector velocity span and $R V$ for individual spectra are listed in Table 1. The trend of the BVS obtained in this work is similar to that of Queloz et al. (2001); the larger value we found for the slope can be attributed to the higher resolution of the SARG spectra.
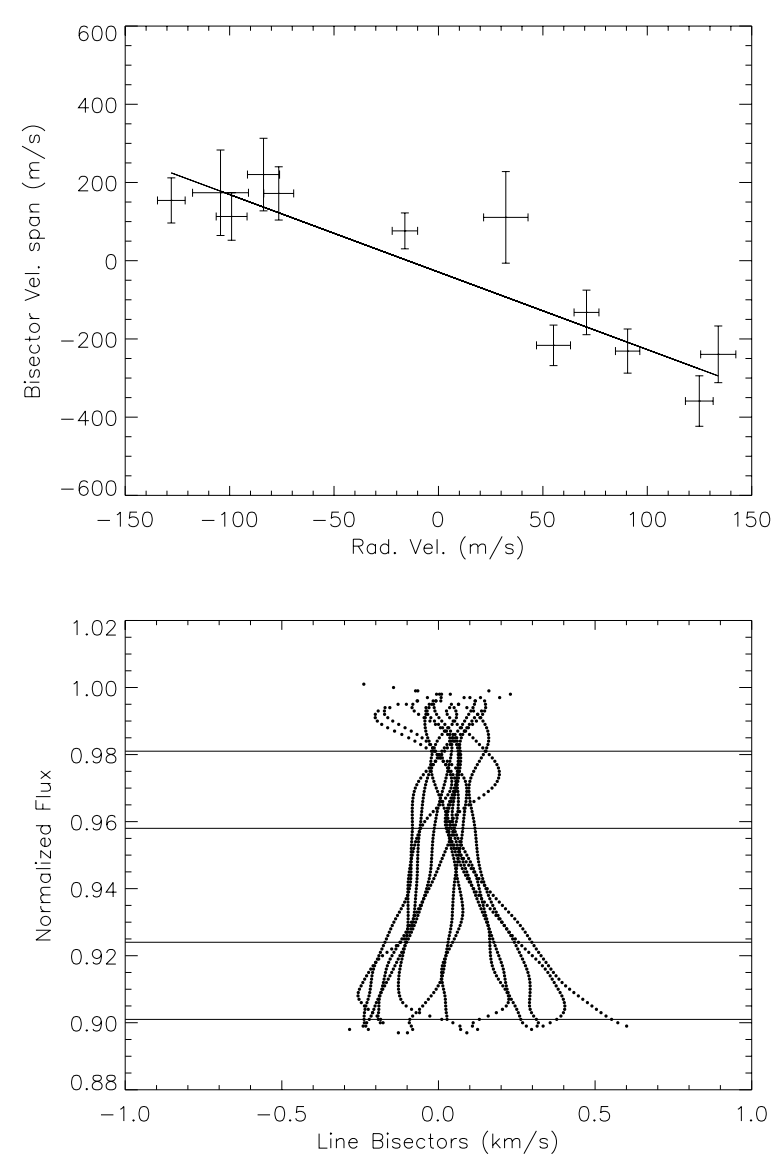

Fig. 6. Upper panel: plot of BVS vs. $R V$ for HD 166435 and the best fit to a straight line. Lower panel: line bisectors for individual spectra adjusted to their corresponding $R V$. The horizontal lines enclose the top and bottom zones considered for the fitting analysis.

\section{2. $H D 8071 B$}

The star HD 8071B of spectral type G0V and visual magnitude $m_{V}=7.6$ is a member of a visual binary system. The primary HD $8071 \mathrm{~A}$ is brighter by $0.3 \mathrm{mag}$ and has a projected separation of 2.1", the smallest in the sample of the SARG survey. Pairs with such small separation are typically not observed in cases of bad seeing; furthermore the slit was oriented perpendicularly to the separation of the components. Nevertheless, some contamination of the spectra is still possible, especially for the secondary component. Our velocity measures show that HD 8071A is itself a single lined spectroscopic binary with semiamplitude of $\sim 7 \mathrm{~km} \mathrm{~s}^{-1}$. The $R V$ difference between the visual components is then variable in the range $(-4,10) \mathrm{km} \mathrm{s}^{-1}$.

Eight spectra of HD $8071 \mathrm{~B}$ were acquired between September 2000 and August 2004. The correlation between BVS and $R V$ found for all spectra has rank correlation coefficient of 0.81 and significance of $98.5 \%$. The analysis of the IP did not show any critical trend acting on the $R V$ computation, leading us to consider contamination as the cause of the observed correlation. The spectrum with $R V \sim 600 \mathrm{~m} \mathrm{~s}^{-1}$ and very different line profile is likely heavily contaminated by the companion, because of the occurrence of technical problems related to telescope tracking that night. Our study of the line 

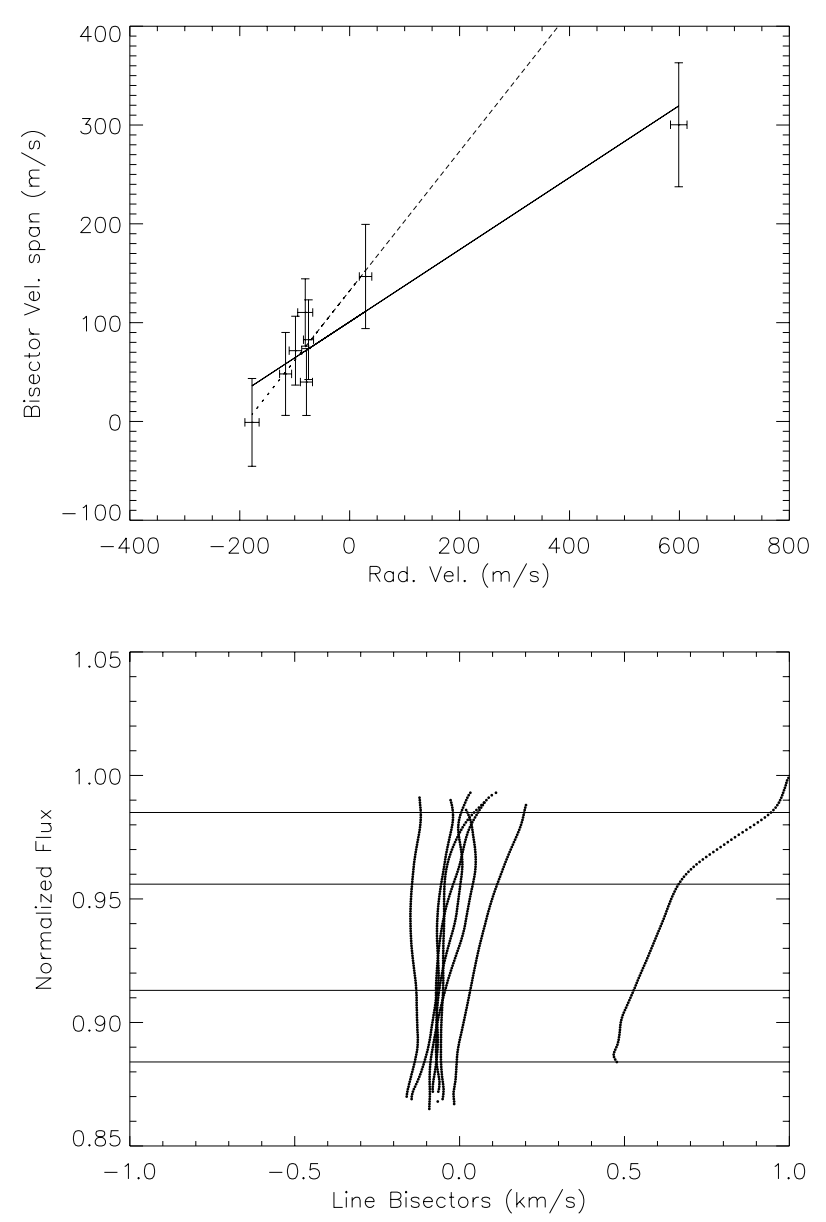

Fig. 7. Upper panel: plot of BVS vs. $R V$ for HD $8071 \mathrm{~B}$ and the best fit to a straight line. The dotted line corresponds to the best fit discarding the spectrum with higher $R V$. Lower panel: line bisectors for individual spectra adjusted to their corresponding $R V$. The horizontal lines enclose the top and bottom zones considered in the fitting analysis.

bisector allows us to clearly identify the problematic spectrum and to exclude it from the analysis of the radial velocity curve.

Nevertheless a correlation still persists even without considering the highly discrepant spectrum, with a rank correlation coefficient 0.71 and significance of $92.9 \%$. This is likely due to some residual contamination (of a few percent at most) by the companion, compatible with the small separation of the pair and the actual observing conditions. Figure 7 shows the two cases and the eight line bisectors computed for all spectra of HD $8071 \mathrm{~B}$, corrected for their $R V$ and plotted on a common reference frame. Values of bisector velocity span and $R V$ for individual spectra appear in Table 2 .

To confirm our hypothesis of contamination as the source of observed BVS- $R V$ correlation, we performed a simple modeling of the expected contamination, excluding the highly discrepant spectrum from JD: 2452982.48. We first determined the light contamination expected on the spectrum of HD 8071B on the basis of the seeing conditions (given by the FWHM of the spectrum measured along a direction perpendicular to the dispersion). $R V$ and BVS of HD 8071B do not show significant correlation with such contamination. We then considered an "effective contamination" as the product of the light

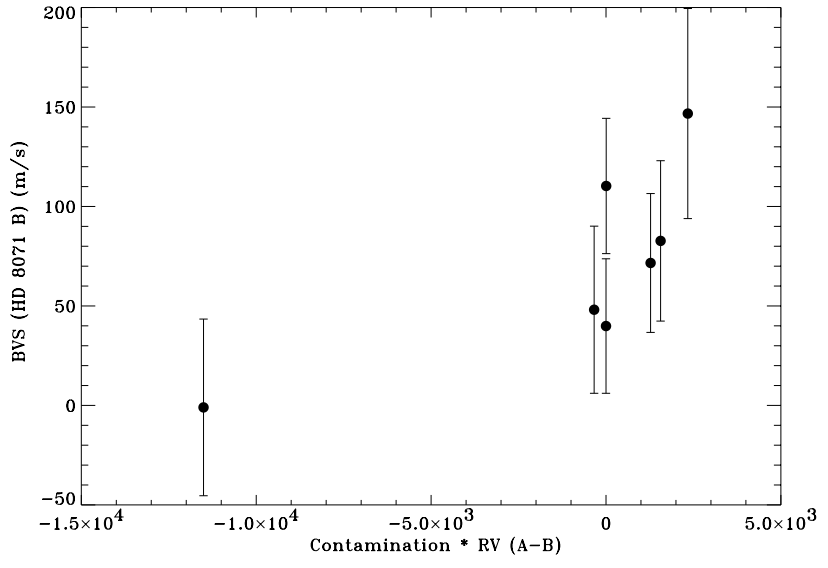

Fig. 8. BVS of HD 8071B vs. the "effective contamination" of $R V$, defined as the product of the light contamination from the companion HD $8071 \mathrm{~A}$ and the $R V$ difference between the components, variable with time because HD 8071A is itself a spectroscopic binary. The highly discrepant spectrum from JD: 2452982.48 was excluded. Abscissa axis has dimensions of (percentage of contamination) $\times$ $(\mathrm{m} / \mathrm{s})$.

Table 2. Bisector velocity span from spectra of HD 8071B. 87 lines were employed in the mask for the CCF.

\begin{tabular}{crrr}
\hline \hline JD - 2450000 & $\begin{array}{c}\left(V_{\mathrm{T}}-V_{\mathrm{B}}\right) \\
\mathrm{m} \mathrm{s}^{-1}\end{array}$ & $\begin{array}{c}V_{r} \\
\mathrm{~m} \mathrm{~s}^{-1}\end{array}$ & $S / N$ \\
\hline 1797.64 & $110.3 \pm 34.0$ & $-80.913 .7 \pm 137$ & \\
1854.54 & $146.7 \pm 52.8$ & $28.8 \pm 11.3$ & 91 \\
2145.71 & $71.6 \pm 34.9$ & $-98.7 \pm 11.4$ & 140 \\
2297.38 & $-1.0 \pm 44.4$ & $-177.7 \pm 12.9$ & 110 \\
2892.61 & $48.1 \pm 42.0$ & $-116.610 .9 \pm 112$ & \\
2982.48 & $300.3 \pm 62.8$ & $598.7 \pm 15.0$ & 92 \\
3216.73 & $39.9 \pm 33.8$ & $-78.7 \pm 11.0$ & 141 \\
3246.68 & $82.7 \pm 40.3$ & $-74.9 \pm 9.2$ & 121 \\
\hline
\end{tabular}

contamination and the variable $R V$ difference between HD $8071 \mathrm{~A}$ and B. The Spearman rank correlation coefficient of $R V$ and BVS vs. such "effective contamination" is 0.85 with a significance of more than $96 \%$, supporting our hypothesis of light contamination as the origin of the $R V$ and BVS variability of HD $8071 \mathrm{~B}$. The relation between $R V$ perturbation and BVS is not linear likely because the $R V$ perturbation due to contamination is expected to be a non-linear function of the position of the contamination across the line profile (see Fig. 8).

\section{3. $51 \mathrm{Peg}$}

The star 51 Peg (HD 217014) of spectral type G2.5IVa and visual magnitude $m_{V}=5.5$ is the first discovered solar-type object to host a planet, with $M_{2} \sin i=0.47 M_{\mathrm{J}}, a=0.052 \mathrm{AU}$ and period of 4.23 days (Mayor \& Queloz 1995).

The star lies in a zone of the Hertzsprung-Russell diagram of stable (very low variability) objects (Eyer \& Grenon 1997). Indeed, according to Henry et al. (2000), no measurable change in mean magnitude (over 5 years) was seen and the Ca II record displayed a signal essentially constant despite some season-toseason jitter and a general indication of a low activity level. 

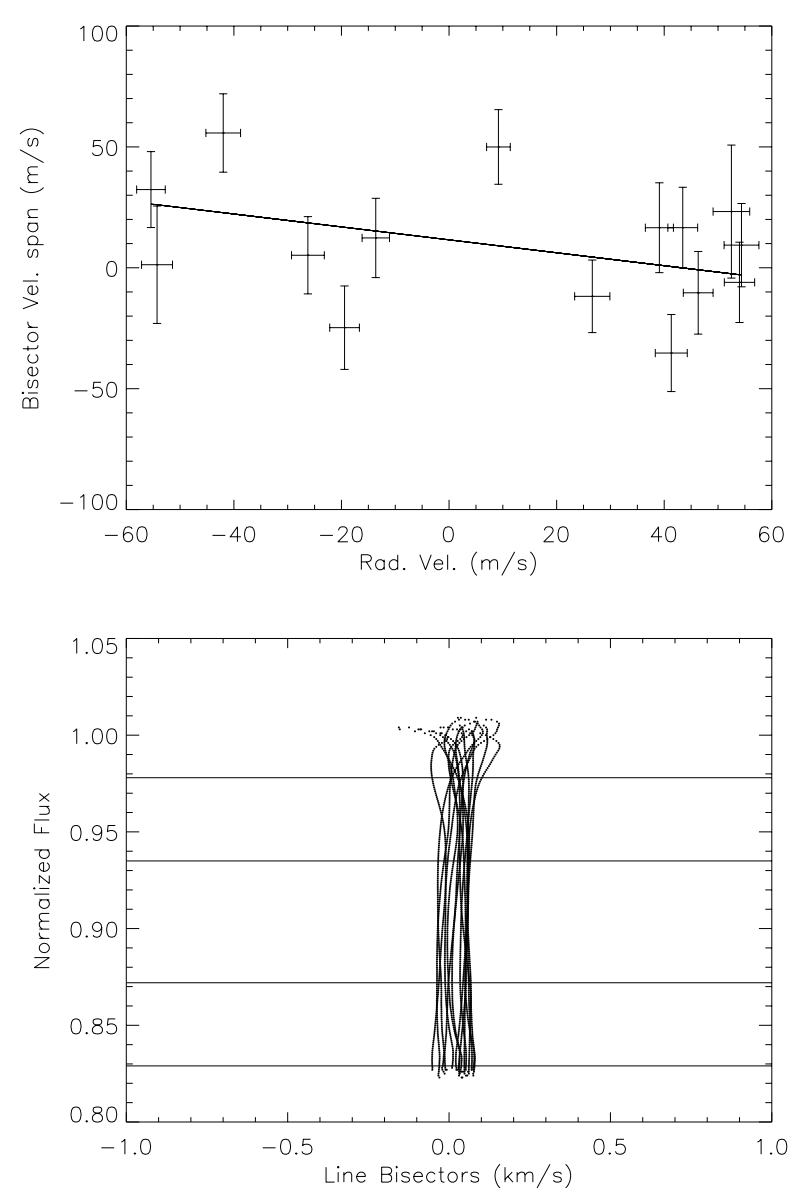

Fig. 9. Upper panel: plot of BVS vs. $R V$ for 51 Peg and the best fit to a straight line. Lower panel: line bisectors for individual spectra adjusted to their corresponding $R V$. The horizontal lines enclose the top and bottom zones considered for the fitting analysis. 74 lines were employed in the mask for the CCF.

Fifteen spectra of 51 Peg were acquired between June 2001 and November 2003. There is no significant correlation between BVS and $R V$ (rank correlation coefficient of -0.28 and significance of $56 \%$ ), confirming the results by Hatzes et al. (1998) and Povich et al. (2001). The line bisector shape (see Fig. 9) seems to be constant.

\section{4. $\rho \mathrm{CrB}$}

The star $\rho$ CrB (HD 143761) of spectral type G0Va and visual magnitude $m_{V}=5.4$ is known to host a planet with $M_{2} \sin i=$ $1.04 M_{\mathrm{J}}, a=0.22 \mathrm{AU}$ and a period of 39.95 days (Noyes et al. 1997).

Twenty six spectra of $\rho \mathrm{CrB}$ were acquired between April 2001 and March 2004. In the plot of BVS against $R V$ the dispersion of the data points shows no correlation (as in Povich et al. 2001) with a rank correlation coefficient of 0.15 and significance of $52 \%$.

The line bisectors and its behaviour, similar to those for $51 \mathrm{Peg}$, are shown in Fig. 10. The typical "C" shape of line bisectors is more evident for $\rho \mathrm{CrB}$ in agreement with the warmer temperature of the star.
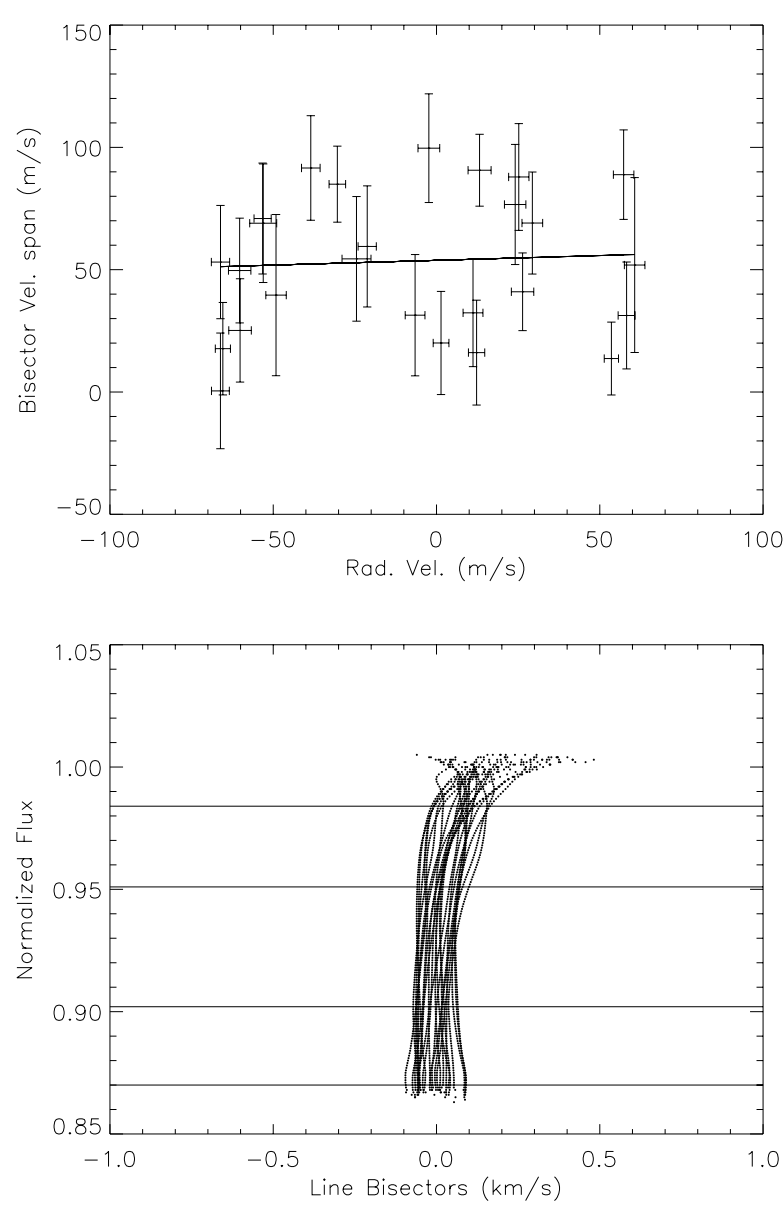

Fig. 10. Upper panel: plot of BVS vs. RV for $\rho \mathrm{CrB}$ and the best fit to a straight line. Lower panel: line bisectors for individual spectra adjusted to their corresponding $R V$. The horizontal lines enclose the top and bottom zones considered for the fitting analysis. 85 lines were employed in the mask for the CCF.

\section{5. $H D 219542 B$}

The star HD 219542B is member of a wide binary system, with spectral type G7V and visual magnitude $m_{V}=8.6$. It was considered in Desidera et al. (2003) as a candidate to host a planet, but ultimately discarded after further analysis; the small $R V$ variations are more likely related to a moderate stellar activity (Desidera et al. 2004a).

For the present analysis of the line bisectors, only the data of the 2002 season were considered, twelve spectra, because the $R V$ scatter and chromospheric activity were greater in this season (see Desidera et al. 2004a). Therefore it should be easier to find a correlation between $\mathrm{BVS}$ and $R V$ from these data alone.

The plot of BVS against $R V$ and the line bisectors are shown in Fig. 11. No correlation appears in this case: the rank correlation coefficient is of -0.37 and the significance of $76 \%$. This lack of correlation is due to the small velocity amplitude (approximately between -17 and $26 \mathrm{~m} \mathrm{~s}^{-1}$ ) and to the low $S / N$ of the available spectra $\sim 100$. 

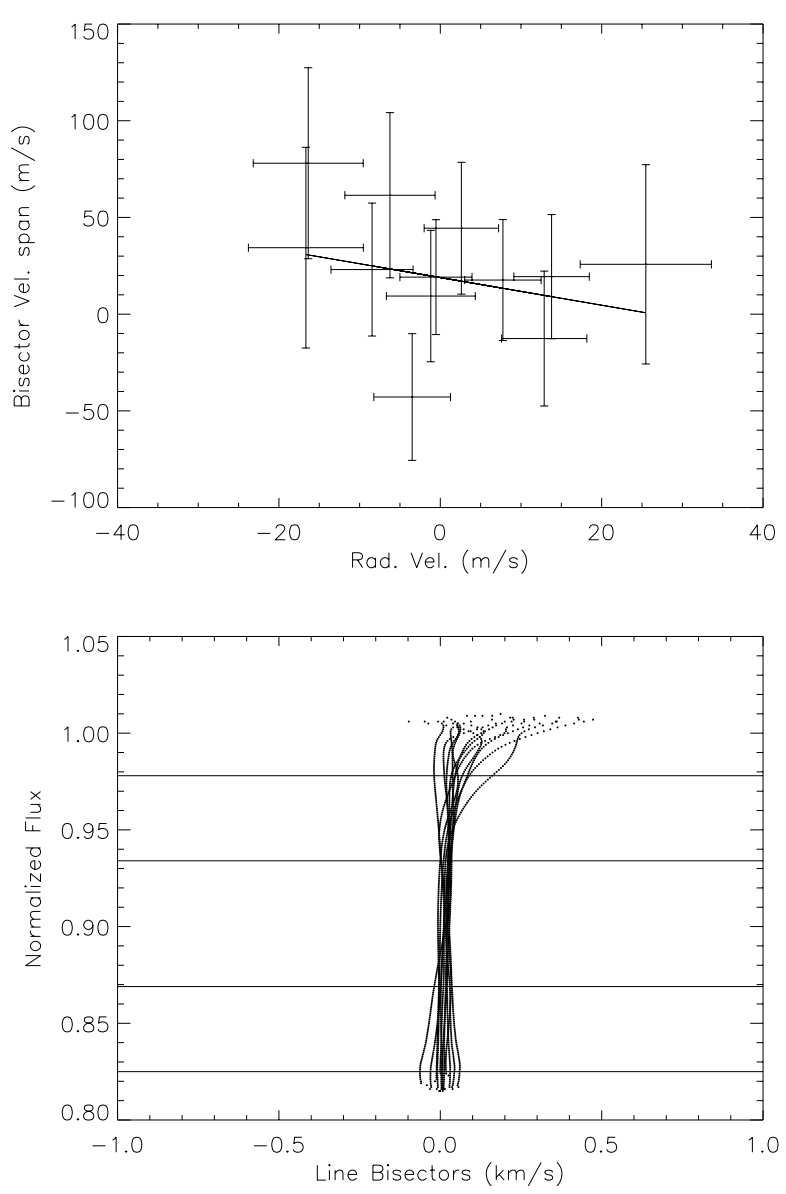

Fig. 11. Upper panel: plot of BVS vs. $R V$ for $\mathrm{HB} 219542 \mathrm{~B}$ and the best fit to a straight line. Lower panel: line bisectors for individual spectra adjusted to their corresponding $R V$. The horizontal lines enclose the top and bottom zones considered for the fitting analysis. 86 lines were employed in the mask for the CCF.

\section{Error analysis}

An analytic study of errors was performed starting by considering the internal BVS errors. The expected errors of BVS were computed for absorption profiles obtained by convolution of Gaussian and rotational profiles. The former were determined with a thermal broadening factor estimated by colors and temperatures of each star and the latter with a $V \sin i$ factor determined by the Fast Fourier Transform (FFT) analysis of each star's absorption profile (Gray 1992).

The observed errors of BVS were the quadratic mean of the error bars of the single spectra. The observed BVS spread was the rms average of the individual BVS for the stars that do not show a significant BVS vs. $R V$ correlation (51 Peg, $\rho \mathrm{CrB}$ and HD 219542B), and the rms of residuals from the linear correlation for HD 166435 and HD 8071B.

The values of errors estimated by these procedures are given in Table 3. The plot in Fig. 12 compares the observed spread of data with expectations based on internal errors alone. These two set of values are fully consistent with each other, indicating that our procedure is not affected by large systematic errors.

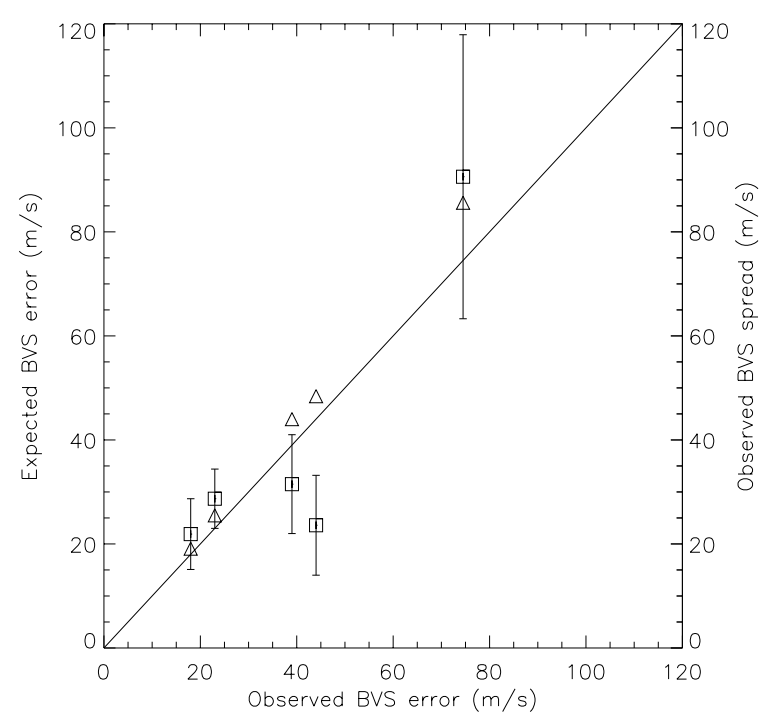

Fig. 12. The expected against observed errors of BVS (triangles: quadratic mean of error bars). Squares correspond to observed BVS spread against the measured BVS errors with spread errors as error bars.

Table 3. BVS errors: computed by Eq. (1) in convolved profiles (expected) and measured from CCF profiles (observed).

\begin{tabular}{lccc}
\hline \hline Star & $\begin{array}{c}\text { BVS error } \\
\text { observed (rms) } \\
\mathrm{m} \mathrm{s}^{-1}\end{array}$ & $\begin{array}{c}\text { BVS error } \\
\text { expected } \\
\mathrm{m} \mathrm{s}^{-1}\end{array}$ & $\begin{array}{c}\text { BVS spread } \\
\text { observed } \\
\mathrm{m} \mathrm{s}^{-1}\end{array}$ \\
\hline HD 166435 & 74.5 & 85.6 & $90.6^{a} \pm 27.3$ \\
HD 8071B & 44.0 & 48.4 & $30.9^{a} \pm 11.7$ \\
51 Peg & 18.0 & 19.1 & $25.3 \pm 6.8$ \\
$\rho$ CrB & 23.0 & 25.5 & $28.7 \pm 5.7$ \\
HD 219542B & 39.0 & 44.0 & $31.5 \pm 9.5$ \\
\hline
\end{tabular}

${ }^{a}$ These values correspond to the rms of residuals from the linear correlation.

\section{Conclusions}

We studied the variation of line bisectors in the same spectra acquired through the iodine cell, employed also for high precision $R V$ measurements. We found that such variation, as measured by the BVS, shows spreads fully consistent with internal errors, as determined from photon statistics, spectral resolution and intrinsic line profiles. We correlated the variation of the bisector span with high precision $R V \mathrm{~s}$ for five stars.

A significant correlation was established in two cases: an anticorrelation for HD 166435, as found by Queloz et al. (2001). This is due to the stellar variability and magnetic activity, which makes the core of the profiles change from positive to negative values of $R V$. A positive correlation for HD 8071B was due to contamination of the spectra by light from the companion star producing an asymmetry in the red wings of the profiles with a consequent inclination of the line bisectors toward positive values of $R V$.

For the stars known to host exoplanets, $51 \mathrm{Peg}$ and $\rho \mathrm{CrB}$, no correlation was found, further supporting the conclusion that in these two cases $R V$ variations are due to Keplerian motion. 
The special case of HD 219542B shows that the bisector technique does not allow us to disentangle the activity origin of low amplitude $R V$ variations $\left(\sim 20 \mathrm{~m} \mathrm{~s}^{-1}\right)$ using spectra of $S / N \sim 100$ even at the high resolution of SARG spectra $(R \sim$ $150000)$.

We conclude that spectra acquired using the iodine cell may be used to study variations of line bisectors. In order to achieve the required accuracy, it is necessary to deal with high quality spectra, in particular high $S / N$ to reduce the error bars in the $\mathrm{BVS}$, or to study spectra where the $R V$ variations are of large amplitude.

Acknowledgements. We thank the referee Dr. Steven H. Saar for his very detailed report that helped to improve the paper. A. F. Martínez Fiorenzano thank Mikhail Varnoff for support and advice. This work was partially funded by COFIN 2004 "From stars to planets: accretion, disk evolution and planet formation" by Ministero Università e Ricerca Scientifica Italy.

\section{References}

Beckers, J., Bridges, C., \& Gilliam, L. 1976, Atlas of the Solar Flux Spectrum 3800-7000 Å, Sacramento Peak Solar Observatory, DOC. AFGL-TR-76-0126

Butler, R. P., Marcy, G. W., Williams, E., et al. 1996, PASP, 108, 500

Desidera, S., Gratton, R. G., Endl, M., et al. 2003, A\&A, 405, 207

Desidera, S., Gratton, R. G., Endl, M., et al. 2004a, A\&A, 420, L27

Desidera, S., Gratton, R. G., Claudi, R. U., et al. 2004b, in Extrasolar Planets Today and Tomorrow, ASP Conf. Ser., 321, 103

Dravins, D., Lindegren, L., \& Nordlund, Å. 1981, A\&A, 96, 345

Endl, M., Kürster, M., \& Els, S. 2000, A\&A, 362, 585

Eyer, L., \& Grenon, M. 1997, ESA SP-402, 467
Gratton, R. G., Bonanno, G., Bruno, P., et al. 2001, Exp. Astron., 12, 107

Gray, D. F. 1982, ApJ, 255, 200

Gray, D. F. 1983, PASP, 95, 252

Gray, D. F. 1988, Lectures on Spectral-Lines Analysis: F, G and K stars (Arva, ON: The Publisher)

Gray, D. F. 1992, The Observation and Analysis of Stellar Photospheres (Cambridge: Cambridge University Press)

Hatzes, A., Cochran, W. D., \& Bakker, E. J. 1998, ApJ, 508, 380

Hatzes, A. 2002, Astron. Nachr., 323, 392

Hill, G. 1982, Publ. Dominion, Ap. Obs., 16, 67

Henry, G. W., Baliunas, S. L., Donahue, R. A., et al. 2000, ApJ, 531, 415

Kürster, M., Endl, M., Rouesnel, F., et al. 2003, A\&A, 403, 1077

Martínez Fiorenzano, A. F. 2005, Ph.D. Thesis, in preparation

Mayor, M., \& Queloz, D. 1995, Nature, 378, 355

Moore, C. E., Minnaert, M. G. J., \& Houtgast, J. 1966, The solar spectrum $2935 \AA$ to $8770 \AA$ (National Bureau of Standards Monograph, Washington: US Government Printing Office, USGPO)

Noyes, R. W., Jha, S., Korzennik, S. G., et al. 1997, ApJ, 483, L111

Paulson, D., Saar, S. H., Cochran, W. D., \& Henry, G. W. 2004, AJ, 127,1644

Povich, M. S., Giampapa, M. S., Valenti, J. A., et al. 2001, AJ, 121, 1136

Queloz, D., Henry, G. W., Sivan, J. P., et al. 2001, A\&A, 379, 279

Saar, S. H., \& Donahue, R. A. 1997, ApJ, 485, 319

Saar, S. H., Butler, R. P., \& Marcy, G. W. 1998, ApJ, 498, L153

Saar, S. H. 2003, in Scientific Frontiers in Research on Extrasolar Planets, ed. D. Deming, \& S. Seager, ASP Conf. Ser., 294, 65

Toner, C. G., \& Gray, D. F. 1988, ApJ, 334, 1008

Torres, G., Konacki, M., Sasselov, D. D., et al. 2004, ApJ, 614, 979

Torres, G., Konacki, M., Sasselov, D. D., et al. 2005, ApJ, 619, 558 